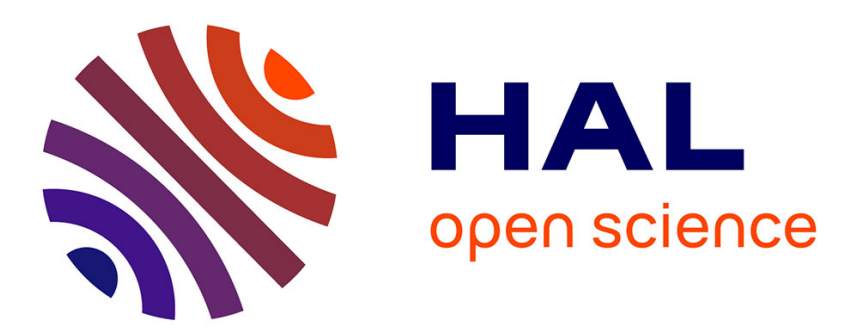

\title{
Les voyageurs occidentaux à la découverte de l'altérité musulmane au bas Moyen Âge \\ Nissaf Sghaïer
}

\section{To cite this version:}

Nissaf Sghaïer. Les voyageurs occidentaux à la découverte de l'altérité musulmane au bas Moyen Âge : L'exemple de Bertrandon de la Broquière. Frontière $\times \mathrm{s}$ : revue d'archéologie, histoire et histoire de l'art, 2019, 1, 10.35562/frontieres.103 . hal-02949660

\section{HAL Id: hal-02949660 \\ https://hal.science/hal-02949660}

Submitted on 29 Sep 2020

HAL is a multi-disciplinary open access archive for the deposit and dissemination of scientific research documents, whether they are published or not. The documents may come from teaching and research institutions in France or abroad, or from public or private research centers.
L'archive ouverte pluridisciplinaire HAL, est destinée au dépôt et à la diffusion de documents scientifiques de niveau recherche, publiés ou non, émanant des établissements d'enseignement et de recherche français ou étrangers, des laboratoires publics ou privés. 


\title{
Frontière's \\ Revue d'archéologie, histoire et histoire de l'art
}

\author{
Publiée avec le soutien de \\ la Maison de l'Orient et de la Méditerranée Jean Pouilloux \\ dans le cadre de la pépinière de revue de \\ l'Université JeAn MOULIN Lyon III
}

\section{$\mathbf{N}^{0} 1$ \\ Dépasser la frontière}

dir. Vincent CHOLLIER et Gaëlle PERROT 


\section{Frontière $\cdot s$}

Maison de l'Orient et de la Méditerranée

5/7 rue Raulin

69365 LYON CEDEX 07

frontiere-s@mom.fr

Pour soumettre un article et consulter l'appel en cours :

www.frontiere-s.mom.fr

https://publications-prairial.fr/frontiere-s

Directrice des publications

Françoise LE MORT, directrice de la MOM

Rédacteurs en chef

Fabien BIÈVRE-PERRIN (IRAA)

Vincent CHOLLIER (HiSoMA)

Gaëlle PERROT (HiSoMA)

\section{Comité de rédaction}

Loubna AYEB (Archéorient)

Mathilde DURIEZ (ArAr)

Cécile MOULIN (HiSoMA, ArAr)

\section{Secrétaire de rédaction}

Vincent CHOLLIER (HiSoMA)

\section{Direction du numéro}

Vincent CHOLLIER er Gaëlle PERROT (HiSoMA)

\section{Autrices}

Stéphanie ANTHONIOZ (Université catholique de Lille, Orient \& Méditerranée UMR 5167)

Marine LÉPÉE (Université Lumière Lyon 2, ArAr UMR 5138, Université de Lausanne, Institut d'Archéologie et des Sciences de l'Antiquité $\mathrm{CH}$ )

Élise PAMPANAY (Université Lumière Lyon 2, HiSoMA UMR 5189)

Nissaf SGHAÏER (Université Saint-Louis Bruxelles, Centre de recherches en histoire du droit, des institutions et de la société) Anne TICHIT (HALMA UMR 8164)

\section{Photo de couverture :}

Coupe de type A attique, Richmond (Virginia), Museum of Fine Arts, inv. 62.1.11, vers 520

Source : https://www.vmfa.museum/piction/6027262-182968811/ (CC-BY-NC) 


\section{Sommaire du numéro}

Mythological Crossings in Ancient Near East

Gilgameš and Ezekiel 47

Stéphanie Anthonioz

5

Les boutiques Dans Les COLONIES De Lyon et DE VIENNE ENTRE LE I ${ }^{\mathrm{ER}}$ S. AV. ET LE III ${ }^{\mathrm{E}}$ S. APR. J.-C. Franchir le seuil, dépasser la frontière

Marine Lépée

DiALOGUES D’ÉPOUX SUR LES STÈLES FUNÉRAIRES ATTIQUES

Dépasser la frontière de la mort

Élise Pampanay

LES VOYAGEURS OCCIDENTAUX À LA DÉCOUVERTE DE L'ALTÉRITÉ MUSUlMANE AU BAS MoYEN ÂGE L'exemple de Bertrandon de la Broquière

Nissaf Sghaïer

LE RENOUVELLEMENT DU RÉPERTOIRE ATTIQUE PAR EMPRUNTS

L'inspiration au-delà des frontières

Anne Tichit 


\title{
Les voyageurs occidentaux à la découverte de l'altérité musulmane au bas Moyen Âge \\ L'exemple de Bertrandon de la Broquière \\ DOI : $10.35562 /$ frontieres. 103
}

\begin{abstract}
Nissaf Sghaïer
Assistante chargée d'enseignement et doctorante, Université Saint-Louis Bruxelles, Centre de recherches en histoire du droit, des institutions et de la société
\end{abstract}

\begin{abstract}
Résumé. $\quad$ En 1432, Bertrandon de la Broquière, premier écuyer tranchant du duc de Bourgogne Philippe le Bon, est envoyé par ce dernier en mission de reconnaissance en Orient. De ce périple qui l'amènera à parcourir par voie terrestre le Proche Orient, l'Asie Mineure, les Balkans et l'Europe centrale, Bertrandon rédigera, à la demande du duc, un récit intitulé Le Voyage d'Outremer. Cette relation se distingue par la richesse des descriptions proposées à partir des observations et des discussions de Bertrandon durant son voyage. Elle constitue donc une source non négligeable en termes de connaissance et de perception de l'altérité musulmane pour un noble bourguignon de la première moitié $d u x v^{e}$ siècle. Le présent article propose d'analyser comment Bertrandon de la Broquière a réussi à se rapprocher des diverses sociétés musulmanes rencontrées pour en proposer une description qui dépasse les stéréotypes ambiants de son époque.
\end{abstract}

Mots-clés : Pays-Bas Bourguignons, voyage, Orient, Turc, altérité, perception

\begin{abstract}
In 1432, Bertrandon de la Broquière, the first Sharp Squire of the Duke of Burgundy Philippe le Bon, was sent in the Middle East on a reconnaissance mission. At the Duke's request, Bertrandon wrote a travel story from his journey-which led him to travel overland through the Near East, Asia Minor, the Balkans and Central Europe-entitled Le Voyage d'Outremer. This narrative distinguishes itself by the richness of its descriptions, based on Bertrandon's observations and discussions during his trip. It is therefore a significant testimony on knowledge and perception of the Muslim otherness in the first half of the I5th century written by a noble Burgundian. This article analyses how Bertrandon de la Broquière managed to get closer to various Muslim societies he met, in order to convey a description that goes beyond stereotypes.
\end{abstract}

\section{Keywords: Burgundian Netherlands, travel, Middle East, Turkish, otherness, perception}

Dans l'Europe occidentale médiévale, les récits de voyage en Orient constituent un genre littéraire bien répandu. La majorité de ceux-ci offrent une énumération des lieux saints et proposent, quand ils les évoquent, des descriptions stéréotypées des populations musulmanes rencontrées sur place. Certains voyageurs se distinguent néanmoins par des expéditions plus lointaines, mêlant dans leur récit observations réelles et visions imaginaires, comme Jean de Mandeville ou Marco Polo. Cependant, à partir du XIV siècle, certaines relations de voyage témoignent d'un intérêt plus marqué pour les sociétés du Proche-Orient. Cette curiosité peut s'expliquer entre autres par la présence croissante de récits de voyage en Orient rédigés par des auteurs laïcs ${ }^{1}$. À cet égard, Le voyage d'Outremer ${ }^{2}$ de Bertrandon de la Broquière, premier écuyer tranchant du duc de Bourgogne Philippe le Bon, offre des considérations intéressantes. En effet, plus qu'un simple pèlerinage en

$1 \quad$ Rouxpetel 2015, p. 27.

2 L'édition utilisée est celle de Schefer C. et Cordier H., publiée en 1892. 
Terre Sainte, le voyage de Bertrandon constitue une mission d'information commanditée par Philippe le Bon pour servir ses projets de croisade contre l'expansion de l'Empire ottoman. Cette mission amena l'envoyé bourguignon à parcourir entre février 1432 et juillet 1433 le Proche-Orient, l'Asie Mineure et les Balkans ${ }^{3}$ et à fournir une description relativement fine des réalités contemporaines de ces régions. Son récit, rédigé vingt ans après son retour ${ }^{4}$, a été conservé dans la bibliothèque des ducs de Bourgogne ${ }^{5}$.

Les nombreuses considérations fournies sur les sociétés musulmanes rencontrées témoignent d'une approche différente de celle d'autres voyageurs de la même époque et du même milieu ${ }^{6}$ l'itinéraire particulier emprunté par Bertrandon offre au lecteur des représentations de régions relativement peu connues. Les descriptions des populations rencontrées sont nombreuses, détaillées et concernent des aspects variés, du fonctionnement politique à la vie quotidienne.

Riche de cet intérêt, cette relation de voyage a déjà fait l'objet de nombreuses études, d'autant plus que la littérature viatique médiévale rencontre un nouvel engouement dans l'historiographie récente ${ }^{7}$. Cet article propose d'aborder un aspect du récit qui, à ma connaissance, n'a pas encore fait l'objet d'une publication à part entière, à savoir les procédés par lesquels Bertrandon de la Broquière parvient à « dépasser la frontière » de l'altérité rencontrée - les musulmans, Sarrasins ou Turcs $-{ }^{8}$. En d'autres termes, comment l'envoyé bourguignon parvient-il à mener à bien sa quête de renseignement sur ceux qui étaient considérés depuis plusieurs siècles en Occident comme des ennemis?

\section{RENCONTRES AVEC DES CHRÉTIENS, VECTEURS D'INFORMATIONS}

\section{PRATIQUES}

À l'instar d'autres voyageurs, Bertrandon de la Broquière rencontre des chrétiens occidentaux présents au Proche-Orient sur une plus ou moins longue durée. Les contacts avec les chrétiens d'Orient semblent quant à eux très limités, du moins d'après son témoignage.

\section{Les marchands}

L'envoyé bourguignon relate de nombreux contacts avec des marchands chrétiens, essentiellement italiens, espagnols et français. Ces commerçants sont, logiquement, présents dans les grandes villes orientales (Jérusalem, Damas, Beyrouth...). Forts de leurs expériences, ils lui proposent une aide logistique : hébergement, mise en contact avec des muletiers, accompagnement au bazar pour l'aider à se vêtir tel un musulman (cf. ci-dessous), conseils sur le parcours à suivre, etc. Bertrandon explique ainsi : « Adont me conseillay à ung marchant venissien où j'estoie logié qui s'appelloit Paule Barberigo comment je pourroie faire le pellerinage de Nazareth et du mont de Thabor lequel me fist avoir ung moucre pour moy conduire ; lequel me fist habillier ainsy que les Sarazins sont habilliez [...] »'. D'autres partagent avec lui une partie du chemin, le conseillant également sur la tenue à adopter pour voyager plus en sécurité ${ }^{10}$.

Outre ces aides pratiques, les rencontres avec des marchands chrétiens permettent à Bertrandon de s'enquérir de la situation politique des régions dans lesquelles il se trouve. Ainsi par exemple, un marchand de Chypre rencontré à Tarse (Cilicie) lui « dist et conta de l'estat dudit pays, car il y avoit demouré longtemps et parloit tresbien le langaige $\aleph^{11}$. Ces informations ont permis à Bertrandon de se déplacer en relative sécurité.

3 Bertrandon de la Broquière choisit de rentrer en France par voie terrestre, traversant ainsi l'Asie Mineure et les Balkans, alors que la solution généralement adoptée était de rentrer en bateau en traversant la Méditerranée.

4 Il est intéressant de constater qu'aucune actualisation de la situation en Orient n'a été apportée lors de la rédaction de la relation malgré ce décalage de vingt ans.

5 La composition de cette bibliothèque témoigne de l'intérêt des Ducs de Bourgogne pour l'Orient. L'inventaire dressé en 1467 atteste d'ailleurs la présence d'une rubrique « Outremer, Médecine et Astrologie ». (Doutrepont 1909, p. 263.)

6 À titre d'exemple, Guillebert de Lannoy, un autre Bourguignon également parti en Orient dans la première moitié du XV ${ }^{\mathrm{e}}$ siècle, ne propose pas de descriptions aussi fines sur le quotidien des Sarrasins et des Turcs. Les itinéraires différents de ces deux voyageurs expliquent également qu'on retrouve bien plus de descriptions des Turcs chez Bertrandon de la Broquière que chez Guillebert de Lannoy.

7 Voir entre autres les travaux de Christiane Deluz, Jaroslav Svátek, Sylvia Cappellini, Jacques Paviot.

8 Dans cet article, l'expression « sarrasin » désigne les Arabes du Proche-Orient, conformément à l'usage qu'en fait Bertrandon dans son récit, à une exception mentionnée ci-dessous. Ne seront donc prises en compte ni l'altérité chrétienne orientale (nous renverrons pour cet aspect aux travaux de Camille Rouxpetel) ni l'altérité juive (à peine évoquée dans le récit de Bertrandon de la Broquière).

9 Schefer et Cordier 1892, p. 40-41. Voir aussi p. 44 ; p. 60 ; p. 77.

10 Ainsi : «Je me party de ceste ville de Bourse en la compaignie de trois marchans Jennevois qui menoient leursdites espices à Père. Et pour aller plus seurement, me feisrent achepter un rouge chapeau hault et une huvette de fil d'archal, lequel habillement je portay jusques à Constantinoble » (Schefer et Cordier 1892, p. 137).

11 Schefer et Cordier 1892, p. 100. 
En effet, à cette époque, des tensions entre diverses puissances locales au Proche-Orient et en Asie Mineure contribuaient à rendre les routes dangereuses.

\section{Les diplomates}

Les marchands ne sont pas les seuls chrétiens rencontrés par Bertrandon. Il côtoie également des envoyés diplomatiques en déplacement. Il rejoint ainsi deux ambassades : la première commanditée par le nouveau roi de Chypre au pays de Karman ${ }^{12}$ (territoire d'une dynastie turcomane situé en Anatolie centrale) et la seconde menée par Benedic de Fourlino pour le duc de Milan à Péra ${ }^{13}$ (quartier de Constantinople).

La première offre à l'envoyé bourguignon l'occasion d'observer le protocole de cour du pays de Kar$\operatorname{man}^{14}$. Les diplomates lui proposent à l'issue de leur mission, face aux dangers de la route, de le raccompagner $^{15}$. Ce danger de voyager seul par les terres d'Asie Mineure, comme nous le verrons tout au long de cette brève analyse, ressort à plusieurs reprises des rencontres relatées par Bertrandon ${ }^{16}$.

La seconde ambassade, celle menée par les Milanais à Constantinople, permet à Bertrandon de la Broquière, outre d'obtenir des informations relatives à la situation politique de la région, de rencontrer le sultan ottoman dont il dresse le portrait suivant : bien portant, grossier, porté sur les femmes et les jeunes enfants, amateur de chasse et d'oiseaux. Quant à sa politique, les rouages en sont détaillés de manière élogieuse, comme si Bertrandon dissimulait une critique de la situation politique tendue que connaissait le royaume de France à l'époque de son voyage ${ }^{17}$. Ces multiples renseignements découlent d'observations directes et de propos rapportés par d'autres personnes (« et m’a on-t-dit... », formule régulièrement employée dans le récit du Bourguignon $)^{18}$.

\section{Les hommes d'Église}

De manière assez étonnante, alors que Bertrandon se rend en Terre Sainte, son récit n'évoque que très peu de rencontres avec le clergé et les ordres religieux chrétiens installés sur place. Il rencontre néanmoins un prêtre du consul de Venise ${ }^{19}$ à Damas et lui demande de rédiger tout ce qu'il sait sur Mahomet et le Coran afin que ces informations soient ensuite transmises à Philippe le Bon ${ }^{20}$. Celles-ci ne figurent pas dans la relation de voyage. Soulignons ici que le Voyage d'Outremer, même si Bertrandon a régulièrement fréquenté des musulmans, comporte en lui-même très peu d'informations sur leur religion. La nature de sa mission (espionnage politique) explique sans doute ce constat.

\section{Les convertis et les chrétiens d'Orient}

Sur sa route, Bertrandon rencontre Anthoine de Passerot, banni de Famagoste, ancien serviteur du roi de Chypre, qui avait été accusé d'entretenir une relation avec une femme turque lors d'un séjour en Anatolie centrale. Le roi de Karman l'avait de ce fait obligé à renier sa foi chrétienne ${ }^{21}$. Or il affirma à Bertrandon vouloir « s'en venir devers monseigneur le duc et qu'il ne demeureroit point sarrazin et que le mot qu'il avoit dict, c'estoit pour eschivier la mort et qu'il craignoit fort d'estre circoncis et l'attendoit de jour en jour, car son maistre le voulloit $\gg^{22}$. Rien n'indique cependant qu'il souhaite fuir avec le Bourguignon. Cette rencontre témoigne d'une nostalgie des chrétiens convertis pour leur première religion, ce qui n'est pas sans faire écho aux velléités du duc de Bourgogne qui avait commandité le voyage de Bertrandon. Anthoine a par ailleurs rapporté à Bertrandon des informations sur la situation politique de Chypre ainsi que sur les dangers à parcou-

12 Schefer et Cordier 1892, p. 111

13 Schefer et Cordier 1892, p. 167.

14 Schefer et Cordier 1892, p. 111-116.

15 Schefer et Cordier 1892, p. 121.

16 Par exemple deux marchands chypriotes qui proposent à Bertandon de les accompagner dans leur retour en bateau, le retour par terre vers la France étant bien trop dangereux selon eux : « Ilz me dirent que ce seroit chose impossible et que se je avoye mille vies, je les perdroye aincois. » (Schefer et Cordier 1892, p. 107).

17 Svatek 2016, p. 72. Cette technique littéraire sera utilisée dans les siècles postérieurs par certains intellectuels pour critiquer leur société. John Tolan a ainsi souligné que le prophète était, aux $16^{\mathrm{e}}$ et $17^{\mathrm{e}} \mathrm{s}$., généralement mieux perçu, tant aux yeux des papistes que des protestants, que les courants religieux auxquels ces derniers s'opposaient réciproquement (Tolan 2018, p. 150).

18 Schefer et Cordier 1892, p. 181-194. À noter, les fréquentes précisions sur la source d'information (observation directe ou témoignage de seconde main), ce qui n'est pas sans rappeler les Histoires d'Hérodote, témoignent de l'esprit critique appliqué par Bertrandon dans sa quête de renseignements.

19 Aucune précision n'est apportée par Bertrandon sur l'éventuelle appartenance de ce prêtre à un ordre religieux qui « disoit souvent messe à l'ostel dudit consul et confessoit et ordonnoit lesdit marchans en leurs necessitez ». (Schefer et Cordier 1892, p. 58).

20 Schefer et Cordier 1892, p. 58.

21 Schefer et Cordier 1892, p. 117.

22 Schefer et Cordier 1892, p. 119. À noter ici, l'usage du terme Sarrasin pour désigner une personne musulmane, indépendamment de son origine géographique alors qu'à d'autres passages, Bertrandon utilise le terme de Maure pour désigner un musulman. 
rir les routes montagneuses qui mènent vers l'Arménie ${ }^{23}$. De même il lui affirma que le roi de Karman détestait le sultan ottoman ${ }^{24}$, témoignant ainsi des tensions politiques qui traversaient l'Asie Mineure à l'époque.

Si quelques explications sont fournies à leur sujet ${ }^{25}$, aucune relation avec des Chrétiens d'Orient n'est évoquée par Bertrandon, hormis quelques mentions d'un Arménien qui lui fournit des informations sur les régions environnantes ${ }^{26}$. Faut-il y voir une conséquence des tensions de cette époque avec les chrétiens d'Orient? Les propos négatifs formulés à l'égard des Grecs corroborent cette hypothèse. En effet Bertrandon affirme faire plus confiance et éprouver plus d'amitié envers les Turcs qu'envers les Grecs ${ }^{27}$. Ce discours ne semble cependant viser explicitement que les Grecs, non les autres communautés chrétiennes orientales.

\section{Une aide pour mieux connaître l'altérité musulmane ?}

Au fil de son périple, Bertrandon observe que les chrétiens sont « fort haïs $»^{28}$ en Orient. Dès lors, s’ils l'aident, notamment d'un point de vue pratique, ce ne sont pas toujours les meilleurs intermédiaires pour découvrir la société musulmane de plus près, à l'exception des ambassadeurs qui lui ont permis d'approcher les cours politiques.

Ces intermédiaires chrétiens assistent néanmoins Bertrandon dans les aspects pratiques et logistiques de son voyage (dans les villes, il est logé presque toujours chez des marchands chrétiens), lui apportent des informations sur la situation politique des régions traversées et lui permettent de rencontrer des chefs musulmans, dont le sultan ottoman. Par ailleurs, au fil de la lecture du Voyage d'Outremer, il est possible d'identifier un réseau de connaissances regroupant certaines personnes rencontrées par Bertrandon en Orient : par exemple «... le premier Crestien que je rencontray, ce fu celluy à qui Parvezin de Barut m'avoit baillié lettres pour luy porter, lequel estoit des Espignolins de Jennes... $»^{29}$. Cette observation, qui mériterait d'être davantage étudiée, laisse supposer une entre-aide parmi les chrétiens présents au Proche-Orient, entre-aide pouvant faciliter les circulations.

\section{RENCONTRE AVEC LES MUSULMANS : AU-DELÀ DU SIMPLE ESPIONNAGE}

Si le point précédent ne distingue pas spécifiquement le Voyage d'Outremer d'autres récits et n'apporte que des informations limitées pour l'écuyer bourguignon sur le monde musulman, la fréquence et la richesse des moments partagés avec des Sarrasins et des Turcs constituent bien une particularité du récit. Certes d'autres voyageurs témoignent de contacts avec des officiers ou guides musulmans, mais leurs relations semblent demeurer très superficielles, ceux-ci n'étant mentionnés qu'au travers des scènes décrites. À l'inverse, Bertrandon, qui les désigne parfois de $«$ mes guides $»^{30}$, témoigne d'une intimité qui se développe avec certains, citant d'ailleurs le nom de plusieurs de ses compagnons de route.

\section{Les intermédiaires officiels des pèlerins}

Au début de son périple, les rencontres de Bertrandon avec des musulmans se placent dans le cadre d'un traditionnel pèlerinage en Terre Sainte : il s'agit des officiers et traducteurs (truchemens) du sultan mameluk chargés d'accueillir les pèlerins chrétiens fraîchement débarqués à Jaffa. Aucun élément ne permet d'affirmer que Bertrandon ait développé une relation particulière avec eux, d'autant qu'il voyageait à ce moment avec un groupe de pèlerins occidentaux. Néanmoins il cite le nom de deux truchements auxquels il a eu affaire : Nasr Eddin et Saad Allah ${ }^{31}$. Outre ce premier accueil, les pèlerins sont également accompagnés par des Bédouins (Arabes) dans le désert du Sinaï pour accomplir le pèlerinage de Sainte-Catherine. Cette escorte se place dans un cadre organisé et régi par le sultan mameluk.

23 Schefer et Cordier 1892, p. 117-120.

24 Schefer et Cordier 1892, p. 120.

25 Principalement lorsque Bertrandon décrit sa visite en Terre Sainte. Il y évoque alors différentes communautés qui y résident (Schefer et Cordier 1892, p. 11-12). Un Napolitain rencontré à Péra rapportera à Bertrandon plusieurs considérations sur le royaume du prêtre Jean, lesquelles seront intégrées au récit (Schefer et Cordier 1892, p. 142-148).

26 Schefer et Cordier 1892, p. 93.

27 Schefer et Cordier 1892, p. 149. Ces affirmations correspondent aux clichés ambiants de l'époque circulant dans l'Occident chrétien, particulièrement à la cour de Bourgogne. Cette perception négative des Grecs se retrouve déjà chez les chroniqueurs de la première croisade (Rouxpetel 2017, p. 233-235)

28 Schefer et Cordier 1892, p. 22 ; voir aussi p. 38.

29 Schefer et Cordier 1892, p. 131

30 Schefer et Cordier 1892, p. 48.

31 Schefer et Cordier 1892, p. 14-16. À noter, Nasr Eddin, grand truchement de Jérusalem, accompagnera également Pero Tafur, mentionné ultérieurement dans cet article. 
Tombant malade lors de la traversée du désert, Bertrandon se fait soigner par des Bédouins et affirme qu'ils lui ont fait « tresbonne compaignie que ilz n'ont point accoustumé de faire aux Crestiens » ${ }^{32}$. Bertrandon souligne donc assez vite dans son récit qu'il entretient des relations privilégiées avec l'Autre musulman, bien qu'il s'agisse ici d'un groupe en marge du pouvoir du sultan (des tensions entre les Bédouins et le sultan sont mentionnées dans le Voyage d'Outremer) et vivant dans des régions reculées.

\section{Les esclaves}

Au fil du récit, les témoignages de rapprochement avec des musulmans deviennent plus nombreux. Parmi ceux-ci, Bertrandon entretient rapidement des contacts avec des esclaves et des serviteurs, des personnes différentes de lui sur deux aspects : culturellement/religieusement et socialement.

Ainsi, lorsqu'un groupe de pèlerins musulmans, turcs, de retour de La Mecque, arrive à Damas, Bertrandon trouve compagnie auprès d'un des esclaves. Celui-ci lui parle de l'islam, notamment du pèlerinage à la Mecque et du Paradis ${ }^{33}$.

Plus tard, alors qu'il passait la nuit dans un caravansérail de Kütahya, le voyageur se fait voler l'une des courroies de son cheval durant son sommeil. Un esclave, au service du sultan ottoman et que Bertrandon qualifie « d'homme d'auctorité » ${ }^{34}$, voyant qu'il ne parlait pas bien le turc et parlant lui-même italien, lui offre son aide $^{35}$. Cet esclave est probablement un homme d'armes d'origine italienne. Bertrandon le soupçonne en effet d'avoir été capturé à la bataille de Nicopolis ${ }^{36}$. Or, celle-ci ayant eu lieu en 1395 et le voyage de Bertrandon se déroulant 37 ans plus tard, on peut en déduire l'âge avancé de ce captif devenu esclave (qui justifierait le qualificatif d'homme d'autorité ?) et son adaptation dans la société turque, bien qu'aucune information ne confirme une conversion à l'islam. Il lui fournit des conseils sur les provisions à emporter et sur la route à suivre $^{37}$.

Un cas particulier se révèle à la lecture du Voyage d'Oultremer : Bertrandon évoque à maintes reprises un certain « mon mameluk » avec lequel il semble s'être lié d'amitié. Ce dernier, accompagnant une caravane que Bertrandon avait rejointe à Balbeek, s'est rapproché de lui par « charité » en le voyant seul et incapable de parler la langue du pays ${ }^{38}$. Le Bourguignon réalise une grande partie de son périple avec lui, le surnommant « mon mamelu ». Ce surnom peut surprendre le lecteur, d'autant que Bertrandon ne mentionne jamais la présence d'un garde personnel à son service. Il qualifie cet homme dans sa première description de « un des mamelus du Souldan lequel estoit Cerkaisis et aloit pour querir ung sien frere qui estoit au pays de Carman $»^{39}$. Cet esclave faisait donc partie de la garde rapprochée du sultan d'Égypte. Son origine caucasienne n'est pas étonnante puisque les Mameluks étaient essentiellement originaires d'Asie centrale et du Caucase. Formant la caste dirigeante d'Égypte, parmi laquelle le sultan était choisi, leur recrutement dans les régions susmentionnées était régulièrement organisé par les Mameluks eux-mêmes afin de garantir la continuité de leur caste, extrêmement fermée ; aucun arabe ne pouvait en faire partie ${ }^{40}$. Faut-il voir dans ce fonctionnement la raison pour laquelle le susdit mameluk rencontré par Bertrandon allait chercher son frère ? Quant au possessif « mon », il a pu être utilisé par Bertrandon pour souligner la proximité entretenue avec cet homme et/ ou pour le distinguer d'autres mameluks dans son récit.

Quoi qu'il en soit, en sa compagnie, l'envoyé apprend « a couchier sur la terre et à boire de l'eaue sans vin, et me seoir à terre les jambes croisiées, ce qui me fu ung pou dur au commencement $»^{41}$. De même il prend l'habitude de monter à cheval à la turque ${ }^{42}$ et de manger avec une nappe pour ne perdre aucune miette. À cet égard, il peut être intéressant de souligner qu'un siècle et demi plus tard, un ambassadeur européen décrit également l'habitude des Turcs de manger à terre sur une nappe, les accusant de gloutonnerie ${ }^{43}$. Quant à lui, Bertrandon n'émet aucun jugement sur cette manière, mettant au contraire en évidence que cette pratique évite de perdre des miettes du repas. Sa relation s'est développée avec ce mameluk au point que lorsque leurs

32 Schefer et Cordier 1892, p. 23.

33 Schefer et Cordier 1892, p. 57-58

34 Schefer et Cordier 1892, p. 128.

35 Schefer et Cordier 1892, p. 128-129.

36 Schefer et Cordier 1892, p. 129.

37 «Puis me dist qu'il me failloit aller achepter et faire provision pour moy et pour mon cheval pour cinq jours, car je ne trouveroie riens et que je me desjunasse et mengasse de la char, car je n'en avoie point mengié depuis deux jours devant. Et me fit aprez du plaisir beaucop, et me adrecha ad ce que j'avoie à faire » (Schefer et Cordier 1892, p. 129).

38 Schefer et Cordier 1892, p. 70

39 Schefer et Cordier 1892, p. 70.

40 Hourani 1993, p. 164.

41 Hourani 1993, p. 70. Cette description fait écho aux gravures de Pieter Coeck publiées à Anvers en 1533 : Mours et Fachons des Turcs (Paredes 2005, p. 127-128).

42 Si cette technique s'avère au début difficile pour lui, Bertrandon souligne qu'ensuite, elle lui semblera plus aisée que la manière occidentale de monter à cheval.

43 Brizay 2018, p. 42. 
chemins se séparent, il cite son nom, Mahomet, et précise qu'il lui « avoit fait moult de biens. Et ce faisoit il par grant charité [...] Je escrips cecy affin que il me souviengne que ung homme hors de nostre foy, pour l'onneur de Dieu, m'a faict tant de biens. $)^{44}$

Comme souligné précédemment, le danger (vols, attaques, tentatives de meurtre) est une réalité que l'on retrouve à plusieurs reprises dans le récit. Bertrandon relate ainsi que deux Turcs envisageaient de le tuer sous prétexte qu'il était riche, à en croire ses vêtements et ses armes, et qui plus est chrétien. C'est son compagnon mameluk qui le sauva de ce piège ${ }^{45}$. À un autre moment encore il le protégea face à quelques Turcs en lui conseillant de fuir : « et me dist on que se je feusse retourné demie lieu arriere, qu'ilz m'eussent coppé la gorge $[\ldots] \gg{ }^{46}$. Comme l'indiquent ces deux exemples, outre les connaissances que ce compagnon de voyage a apportées à Bertrandon, son rôle de garde rapprochée défini par son statut de mameluk apparaît à plusieurs reprises dans le récit, au bénéfice du Bourguignon.

Les esclaves constituaient donc des intermédiaires en termes d'information sur les réalités orientales et des protecteurs. De manière paradoxale, si le danger est régulièrement souligné par Bertrandon (vol, agression, maladie...), il est aidé, voire sauvé, par des musulmans à plusieurs reprises.

\section{Les autres compagnons de route}

Le fait que Bertrandon se joigne régulièrement à des groupes de musulmans, essentiellement turcs, l'amène à partager avec eux des moments privilégiés. Ceux-ci sont soit des pèlerins de retour de La Mecque, soit des marchands. Ainsi, à plusieurs reprises, son rapprochement avec les Turcs se manifeste autour d'un verre d'alcool dans des tavernes. Citons entre autres exemples des Turcs qui apprennent que l'envoyé bourguignon loge à l'hôtel d'un Franc et lui demandent de leur apporter du vin de cet établissement. Bien que son hôte le lui ait déconseillé pour des raisons de sécurité, Bertrandon se retrouve avec ces Turcs et tous se mettent à boire sans retenue et dans une bonne ambiance ${ }^{47}$. On apprend à cette occasion que l'un des Turcs l'appelle frère (kardays $)^{48}$.

En outre, à l'instar de sa relation avec son mameluk, cette proximité avec des habitants des régions parcourues lui permet de décrire plusieurs aspects de la vie quotidienne : description du caravansérail, des repas (la recette du pain turc est même proposée), etc.

\section{Une aide pour mieux connaître l'altérité musulmane?}

Sur l'ensemble de son récit, Bertrandon entretient davantage de contacts avec des musulmans turcs que sarrasins, ou en tout cas il les souligne davantage. Ce constat peut s'expliquer de plusieurs manières : premièrement, le retour par voie terrestre en passant par l'Asie Mineure et par les Balkans représente une large partie de son périple. De plus, et justifiant cet itinéraire, sa mission avait pour objectif essentiel de ramener au duc de Bourgogne des informations sur les Turcs, plus particulièrement sur la dynastie ottomane. Il est donc normal que l'envoyé bourguignon ait davantage cherché à entrer en contact avec eux. Enfin il est possible, mais cette hypothèse devra être vérifiée à l'appui d'autres sources, que les Turcs soient perçus moins négativement que les Sarrasins par les Occidentaux dans la première partie du $\mathrm{Xv}^{\mathrm{e}}$ siècle. Entretenir des relations plus ou moins amicales avec eux aurait en ce sens pu paraître plus évident qu'avec des Sarrasins ${ }^{49}$. Dès lors, plus que l'altérité sarrasine, c'est l'altérité turque qui est le principal objet du discours de Bertrandon de la Broquière.

Ce constat permet également de souligner une conscience qui apparaît peu à peu en Occident de la diversité du monde musulman : les Sarrasins ne sont pas les seuls représentants de l'islam, et les Turcs sont en réalité divisés en divers beylicats ${ }^{50}$.

Par ailleurs, ces nombreuses rencontres ont permis à l'envoyé de Philippe le Bon d'acquérir de nouvelles connaissances sur les sociétés rencontrées. À cet égard, la majorité des informations rapportées dans le récit ne semblent pas pertinentes pour une quelconque préparation de croisade. Ces rencontres offrent plutôt des occasions de récolter des informations qu'on pourrait qualifier d'ethnographiques. Bertrandon semble ainsi avoir dévié de sa mission première, ce qui pourrait constituer une explication à la publication tardive, et sans modernisation, de son récit.

44 Brizay 2018, p. 121

45 Brizay 2018, p. 71.

46 Brizay 2018, p. 104.

47 Brizay 2018, p. 79-80. Dans le Voyage d'Outremer, les Turcs sont plusieurs fois présentés comme des personnes portées sur la boisson (voir également p. 108), contrairement aux Sarrasins. L'un d'eux (More) reproche d'ailleurs en personne au sultan ottoman sa consommation de vin, contraire aux commandements de Mahomet (Brizay 2018, p. 184).

48 Brizay 2018, p. 80.

49 L'image que fournit Bertrandon des Sarrazins est en ce sens clairement négative, contrairement à la manière dont il dépeint le Turc, bien qu'il le critique ouvertement dans certains passages. Peut-être la conversion plus récente des Turcs à l'islam explique-t-elle cette différence de perception?

50 Vigliano 2017, p. 335-336. 


\section{Se faIRE PASSER POUR UN LOCAL}

À son arrivée à Damas, Bertandon se fait attaquer par des habitants, car il est immédiatement reconnu comme un étranger chrétien. Il décide ensuite de revêtir les habits locaux pour éviter que ce genre de situation ne se reproduise et effectue la majeure partie de son voyage en s'habillant tel un Sarrasin ou un Turc, pratique qui n'apparaît pas si étrange à en croire les propos du Bourguignon. En effet il précise que ce " déguisement » des chrétiens est autorisé par le sultan mameluk pour favoriser leur sécurité ${ }^{51}$. L'envoyé bourguignon demande également à rejoindre une caravane de pèlerins turcs pour remonter avec eux, par voie terrestre et en toute sécurité, vers la ville de Bursa, prétextant qu'il souhaitait y retrouver son frère. Le chef de la caravane l'accepte à condition qu'il s'habille comme eux ${ }^{52}$. Il est alors amené par un commerçant chrétien, Jehan

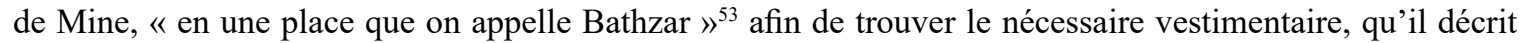
minutieusement dans son récit ${ }^{54}$. Revêtir une tenue locale semble donc bien être un gage de sécurité pour les chrétiens en déplacement à l'est de la Méditerranée.

Il est assez étonnant de remarquer qu'alors que cette disposition semble bien présente en Orient et acceptée par le pouvoir local, Bertrandon est un des seuls, avec Pero Tafur ${ }^{55}$, à stipuler explicitement dans son récit avoir adopté cette stratégie. Ce constat peut s'expliquer par le fait que le statut de pèlerin accordait une forme de protection (cf. encadrement chapeauté par le sultan mameluk) et qu'une grande partie des voyageurs en Orient pouvaient dès lors s'en contenter. Soulignons cependant que quelques décennies plus tard, lors de conquêtes ottomanes, des chrétiens se sont enfuis en se déguisant en Turcs pour échapper à la mort ou à l'esclavage ${ }^{56}$.

Outre cette sécurité, revêtir ces habits locaux offrait à Bertrandon la possibilité de visiter plus facilement les mosquées. En effet, plusieurs récits de voyageurs témoignent de l'interdiction qui leur était adressée de les visiter, principalement celles édifiées sur d'anciens lieux saints chrétiens. Ogier d'Anglure, qui effectua son voyage en 1395, rapporte à propos du site du Temple de Salomon, où s'élève la mosquée Al Aqsa, « ... on n'en peut veoir que l'entrée, et si es ce de bien loing, par dessoubz une volte, c'est assavoir une rue voltée ; et encore ne voit il pas icelle entrée qui veult. Car tantost que les Sarrasins voient venir un chrestien par ycelle rue venant au saint Temple, ilz le rescrient et le font retourner... $\nu^{57}$. De passage à Hébron, Bertrandon témoigne des mêmes difficultés d'accès : « ... car nul Crestien n'ose entrer dedans lesdictes musquées sur peyne de mort ou de renoyer la foy catholicque, et pour ceste cause, nous n'y entrasmes point $\eta^{58}$. Si ces deux exemples concernent d'anciens lieux saints chrétiens convertis en mosquée, une étude systématique croisant ces témoignages avec les textes de lois musulmans permettrait de préciser les sites religieux interdits aux chrétiens et la durée de cette interdiction.

Quoi qu'il en soit, les compagnons de route musulmans de Bertrandon semblent complices de sa duperie, ce qui tend à souligner une nouvelle fois une certaine intimité avec eux : « ... et a illec une belle musquée en laquelle je entray avec mondit moucre, faignant que j'estoie Sarazin $»^{59}$. Cependant, Bertrandon ne fournit aucun détail de ses visites. À titre de comparaison, Guillebert de Lannoy souligne dans son récit la présence de mosquées, ${ }^{60}$ mais sans jamais mentionner qu'il y pénètre.

51 «... car le Souldan a donné congié et licence aux Francs d'aller habilliez en guise de Sarazins pour leur seureté... », Schefer et Cordier 1892, p. 41. Soulignons ici que les Chrétiens, du moins ceux vivant en Orient, devaient généralement revêtir des habits distinctifs. Cette mesure peut varier d'une région à une autre et d'un régime politique à un autre et son application concrète est probablement plus nuancée que les textes juridiques musulmans. Le $\mathrm{XV}^{\mathrm{e}}$ siècle mameluk constitue cependant une période de durcissement envers les dhimmis (Bearman P.J. et al. 1965, p. 236).

52 Schefer et Cordier 1892, p. 60.

53 Schefer et Cordier 1892, p. 60.

54 «... y achetay ce qui m'estoit necessaire touchant cela, c'est assavoir deux robes blanches longues jusques au pié et la tocque de toile acomplie, une courroye de toile et unes brayes de fustenne pour ployer ma robe dedans et ung petit tappis pour couchier sur, unes besaches pour mettre mes choses dedans, unes besaches pour pendre aux oreilles de mon cheval pour mengier son orge et sa paille. Et feis faire ung paletot de panne blanche, lequel je feis tout couvrir de toille qui me fu après tresproffitable de nuyt. Et puis allay acheter ung tarquois tout blanc, tresbien garny. Je achetay aussy une espée et des cousteaulx pour y pendre, à leur guise, ung cullier et une saliere de cuir. ", Schefer et Cordier 1892, p. 60. On remarque au fil de son récit que Bertrandon a apporté une attention particulière aux descriptions des vêtements des Bédouins, des Sarrasins et des Turcs (voir également p. 49 ; p. 77 ; p. $217-220$ ).

55 Voyageur andalou (ca. 1410-ca. 1484). Ne pouvant visiter la mosquée d'Omar à Jérusalem, il décida se vêtir à la manière locale afin d'y rentrer sans encombre. À noter que pour ce dernier, le vêtement du musulman pouvait sembler plus familier puisqu'il était originaire d'Andalousie.

56 Ricci 2007, p. 40-41.

57 Bonnardot et Longnon 1878, p. 41.

58 Schefer et Cordier 1892, p. 18

59 Schefer et Cordier 1892, p. 51-52.

60 Serrure 1840 , p. 70 ; p. 72 ; p. 94. 
Cet habit turc est porté par l'envoyé de Philippe le Bon jusqu'à son retour en France et aurait ainsi contribué à la mode d'influence orientale à la cour de Bourgogne ${ }^{61}$. À cet égard, il relate qu'il éprouve des difficultés à se loger à Vienne, car les gens le prenaient pour un $\operatorname{Turc}^{62}$.

Outre l'habit, Bertrandon a été amené à quelques fois inventer une histoire pour assurer sa sécurité lors de ces rencontres avec des musulmans : comme mentionné ci-dessus, il a prétexté devoir se rendre chez son frère à Bursa. Il s'est aussi fait passer pour un caucasien (cerchais) nouvellement arrivé en territoire turc. Ainsi son mameluk de le présenter à deux personnes qui les hébergent à Eregli : « ... il leur avoit répondu que j'estoye Cerchais et que je ne savois point encores parler le arabich $»^{63}$.

Enfin, Bertrandon observe et surtout adopte des habitudes turques : manière de dormir, de manger, de tirer à l'arc, etc. Cette attitude lui permet de mieux appréhender et décrire les sociétés rencontrées.

Cette stratégie de dissimulation semble bien fonctionner : alors qu'il réalise une partie de son périple seul vers Bursa, des gens, le prenant pour un Turc de retour de La Mecque, lui baisent la main et la robe ${ }^{64}$. Autre exemple, lorsqu'il monte dans un bateau de Grecs à Péra, il est pris pour un Turc et traité avec beaucoup d'honneur. Cependant, une fois que les Grecs découvrent son subterfuge, ils essaient d'obtenir plus d'argent pour le passage ${ }^{65}$. Faut-il déduire de cette anecdote qu'auprès des Grecs, il est plus sûr pour un voyageur d'être turc que chrétien ? Les tensions entre chrétiens occidentaux et grecs à cette époque doivent à nouveau être prises en considération à la lecture de cet épisode.

Quoi qu'il en soit, cette proximité créée avec certains musulmans a permis à Bertrandon d'observer ces sociétés musulmanes au plus près et de fournir à son lecteur des informations obtenues bien souvent de première main.

\section{LES « PRÉMICES ॥ D'UN ORIENTALISME ?}

Plusieurs chercheurs ont déjà souligné que le mouvement orientaliste, tel qu'étudié et décrit par Edward Saïd, trouve ses sources auprès des penseurs des $\mathrm{XVI}^{\mathrm{e}}$ et $\mathrm{XVII}^{\mathrm{e}}$ siècles, lorsque l'Europe se définit elle-même comme une entité à part distincte de l'Orient ${ }^{66}$. C'est également à partir de cette époque que les études sur le monde oriental prennent une nouvelle ampleur, en témoigne la création de la première chaire d'arabe, en 1539, au collège de France ${ }^{67}$.

Cependant, il est possible de trouver les racines de cette curiosité intellectuelle dans les sources tardo-médiévales. En effet, si on est encore bien loin de l'émergence d'un orientalisme académique, le discours de Bertrandon témoigne d'un intérêt - certes politico-militaire dans un premier temps - pour le monde oriental. Si on y retrouve (déjà) certains stéréotypes d'usage chez les orientalistes postérieurs (fourberie, ivrognerie, hypocrisie, perversion sexuelle, lascivité, goût du luxe... $)^{68}$, la Méditerranée orientale semble en même temps devenir pour Bertrandon de la Broquière un objet ethnographique observé à travers son prisme occidental. L'envoyé bourguignon utilise en effet régulièrement la comparaison avec des réalités bourguignonnes dans ses descriptions du monde oriental : le bruit du varan est comparé à celui du chat et sa tête à celle d'un lièvre ${ }^{69}$, les moments de prière sont appelés des heures ${ }^{70}$, les chefs de clan locaux - bey - sont qualifiés de seigneur ${ }^{71}$, les bœufs et les buffles sont utilisés pour transporter les marchandises comme les chevaux en Occident ${ }^{72}$, le yaourt est comparé au lait caillé, ${ }^{73}$ etc. Ce choix stylistique rend la description plus concrète et rejoint la théorie régulièrement avancée que la représentation de l'Autre est influencée par son appartenance socioculturelle $\mathrm{e}^{74}$

61 Coman 2007-2008, p. 106.

62 Schefer et Cordier 1892, p. 239

63 Schefer et Cordier 1892, p. 105.

64 Schefer et Cordier 1892, p. 131

65 Schefer et Cordier 1892, p. 148.

66 Hentsch 1988, p. 78-80.

67 Laurens, Tolan et Veinstein, 2009, p.258.

68 Dès le $\mathrm{xv}^{\mathrm{e}}$ siècle, les représentations du Turc dans les cours occidentales, sur base de ces caractéristiques, en proposent soit une image exotique (habillement luxueux, physique robuste, etc.) soit une image menaçante, rappelant la menace ottomane. (Bost 2005, p. 115-116.)

69 Schefer et Cordier 1892, p. 21-22.

70 Schefer et Cordier 1892, p. 96

71 Schefer et Cordier 1892, p. 87 par exemple.

72 Schefer et Cordier 1892, p. 86.

73 Schefer et Cordier 1892, p. 89.

74 Voir à ce titre, notamment, les travaux de Norman Daniel et d'Edward Saïd. 
Par ailleurs, cette mission d'espionnage commanditée par le duc de Bourgogne en vue de lancer une nouvelle croisade n'est pas sans rappeler l'idée émise par Edward Saïd que le savoir de l'Occident sur l'Orient permet le pouvoir et le contrôle du premier sur le second ${ }^{75}$.

Enfin, Bertrandon n'est pas le seul espion à s'être fait passer pour un local afin d'accomplir sa mission. Par exemple, l'orientaliste Edward Lane (XIX ${ }^{\mathrm{e}}$ siècle) s'est lié d'amitié avec un Égyptien et, en complicité avec ce dernier, s'est fait passer pour un musulman afin de dresser une description du pays et de ses habitants ${ }^{76}$.

\section{CONCLUSION}

Les informations obtenues par Bertrandon sur les régions traversées sont majoritairement issues de ses observations et de discussions avec les personnes, chrétiennes ou musulmanes, rencontrées sur place. Contrairement à d'autres relations de voyage, celle de Bertrandon comporte très peu de passages repris d'auteurs antérieurs. À cet égard, Marian Coman avait déjà mis en évidence le peu de références littéraires du Bourguignon $^{77}$, ce qui ne permet bien sûr pas d'affirmer qu'il n'a pas été influencé par les stéréotypes ambiants de l'époque.

Bertrandon ne voyage quasiment jamais seul. Son récit oscille entre le « je » et le « nous ». Cependant, à l'inverse d'autres voyageurs au Proche-Orient, il se détache rapidement de son groupe de compagnons embarqués avec lui. Dès lors, le «nous » ne se rattache pas tant à une unité culturelle, mais désigne Bertrandon et son/ses compagnon(s) de route musulman(s).

Sur l'ensemble du récit, on constate également que les témoignages de liens privilégiés avec des personnes originaires des régions visitées se concentrent principalement au Proche-Orient et plus encore en Asie Mineure. Bertrandon est en effet plus concis à propos de l'Europe centrale. Ce constat est logique si l'on se rappelle le but de la mission de Bertrandon.

De plus, force est de constater que la proximité atteinte avec les populations locales a amené l'envoyé bourguignon à proposer davantage d'informations sur le quotidien des Turcs, descriptions bien moins polémistes que celles qu'il fournit sur les Sarrasins et les Grecs ${ }^{78}$. Ses diverses interactions lui ont permis de développer une connaissance non négligeable, touchant à divers aspects : politique, culturel, religieux... Ses rencontres, tant avec des chrétiens qu'avec des musulmans, lui ont permis d'acquérir ces informations et attestent que Bertrandon a dépassé des frontières culturelles et religieuses pour mener à bien sa mission d'espionnage. Néanmoins, les informations qu'il consigne dans sa relation ne relèvent au final pas ou peu du strict espionnage politique. Bertrandon a en ce sens dépassé le cadre de sa mission initiale.

Enfin, pour prolonger cette observation, le Voyage d'Outremer pose un paradoxe : si Bertrandon voyage dans des régions politiquement instables en vue d'une potentielle croisade, il s'y déplace relativement librement et donne l'impression de se prendre de sympathie pour ses ennemis.

78 Probablement influencé par la tradition de représentation des Sarrasins présente dans la littérature occidentale depuis plusieurs siècles. 


\section{BIBLIOGRAPHIE}

\section{Sources anciennes}

BonNARDot F. et LongnON A. (éd.) 1878, Le sainct voyage de Jhérusalem du seigneur d'Anglure, Paris.

SCHefer C. et Cordier H. (éd.) 1892, Le voyage d'Outremer de Bertrandon de la Broquière, Paris.

SERrure C.-P. (éd.) 1840, Voyages et ambassades de Messire Guillebert de Lannoy, chevalier de la Toison d'Or, Seigneur de Santes, Willerval, Tronchiennes, Beaumont et Wahégnies. 13991450, Mons.

\section{Travaux}

Bearman P.J., Bosworth C.E., GibB H.A.R., Lewis B. et VAN Donzel E. 1965, Encyclopédie de l'Islam, Leyde.

Bost M. 2005, « La perception des Turcs aux PaysBas à la Renaissance » in R. Puig de la Bellacasa et A. Servantie, L'empire ottoman dans l'Europe de la Renaissance, Louvain, p. 111-122.

BrizaY F. 2018, « Le voyage de Paris à Constantinople de l'ambassadeur de France auprès du sultan ottoman (XVIe-XVIIIe siècle) », Histoire, économie et société, 37, p. 31-45.

DOUTREPONT G. 1909, La littérature française à la Cour des Duc de Bourgogne : Philippe le HardiJean sans Peur - Philippe le Bon - Charles le Téméraire, Paris.

Coman M. 2007-2008, « Experiencing otherness. Bertrandon de la Broquiere's pilgrimage to Jerusalem (1432)», New Europe College Yearbook 2007-2008, p. 85-120.

Daniel N. 1979, The Arabs and Medieval Europe ( $2^{\mathrm{e}}$ éd.), Londres.
Hentsch T. 1988, L'Orient imaginaire. La vision politique occidentale de l'Est méditerranéen, Paris.

Hourani A. 1993, Histoire des peuples arabes, Paris.

Laurens H., Tolan J. et Veinstein G., 2009, L'Europe et l'Islam, quinze siècles d'histoire, Paris.

Paredes C. 2005, « Du texte à l'image. Les tapisseries de la Conquête de Tunis et les gravures des Mœurs et Fachons des Turcs » in R. Puig de la Bellacasa et A. Servantie, L'empire ottoman dans l'Europe de la Renaissance, Louvain, p. 123-150.

RiccI G. 2007, « Crypto-identities. Disguised Turks, Christians and Jews » in A. Molho, D. Ramada Curto et N. Koniordos, Finding Europe: Discourses on Margins, Communities, Images $\mathrm{ca}$. $13^{\text {th }}-\mathrm{ca}$. $18^{\text {th }}$ Centuries, Oxford, p. 3954.

Rouxpetel C. 2015, L'Occident au miroir de l'Orient chrétien. Cilicie, Syrie, Palestine et Égypte (XIIe-XIVe siècle), Rome.

SAÏD E. 2005, L'orientalisme. L'Orient créé par l'Occident (réed.), Paris.

SvateK J. 2016, « L'idéal du souverain oriental dans le récit de Bertrandon de La Broquière » in A. Marchandise et G. Docquier (éd.), Pays bourguignons et Orient : diplomatie, conflits, pèlerinages, échanges (XIVe-XVIe siècles), Neuchâtel, p. 61-72.

Tolan J. 2018, Mahomet l'Européen. Histoire des représentations du Prophète en Occident, Paris.

Vigliano T. 2017, Parler aux musulmans. Quatre intellectuels face à l'islam à l'orée de la Renaissance, Genève. 